\title{
COMUNICACIÓN REMOTA ENTRE FAMILIARES A TRAVÉS DE LA ROBÓTICA SOCIAL
}

Sara Carrasco-Martínez, M. A. Quispe-Flores, Javier Sevilla-Salcedo, Jaime Gómez-Jiménez, Fernando Alonso-Martín, Miguel A. Salichs

RoboticsLab, Departamento de Ingeniería de Sistemas y Automática, Universidad Carlos III de Madrid

\{sacarras, mquispe, jasevill\}@pa.uc3m.es, 100383149@alumnos.uc3m.es, \{famartin, salichs\}@ing.uc3m.es

\section{Resumen}

En la actualidad una gran parte de las personas de avanzada edad no hacen uso frecuente de las Nuevas Tecnologías de la Información. En este trabajo tratamos de eliminar la fricción que tienen este tipo de personas para comunicarse con sus seres queridos de manera natural a través de los medios tecnológicos. Proponemos una aplicación de comunicación accesible e intuitiva implementada con el robot social Mini. Desarrollamos una interfaz gráfica que permite a los usuarios mantener conversaciones, llamadas y videollamadas mediante una aplicación sencilla y de forma guiada a través del robot Mini. Se utiliza como plataforma de comunicación WhatsApp, una de las aplicaciones más utilizadas, a día de hoy, en el mercado de los dispositivos inteligentes. Se pretende contribuir al cuidado y bienestar de la tercera edad.

Palabras clave: Robótica social; Interacción humano-robot; Comunicación a través de la robótica social; Inclusión digital; Redes sociales inclusivas.

\section{INTRODUCCIÓN}

En la sociedad actual está ocurriendo una brecha digital producida por el constante avance de las Nuevas Tecnologías de la Información y Comunicación (TIC) frente al creciente envejecimiento de la población [5]. Según los datos ofrecidos por el Instituto Nacional de Estadística [7], como se puede apreciar en la gráfica representada en la Fig. 1, al aumentar la edad desciende el uso de los dispositivos inteligentes e Internet produciendo un aislamiento tecnológico de este colectivo.

Según un estudio realizado [9] la utilización de las TIC en las personas de avanzada edad proporciona mayor conexión con la actualidad, fomenta su inclusión social y enriquecimiento personal mejorando su calidad de vida y desarrollando sus habilidades sociales. Este colectivo no hace un uso frecuente de las tecnologías actuales debido a los problemas relacionados con la accesibilidad, uso y adopción. En los últimos años, se ha incrementado el desarrollo de dispositivos accesibles e intuitivos con el objetivo de fomentar la e-inclusión en las personas de la tercera edad, concepto entendido como la capacidad de acceder de forma habitual y sencilla a diferentes programas y realizar aprovechamiento de sus utilidades teniendo en cuenta las necesidades específicas de cada usuario [2].

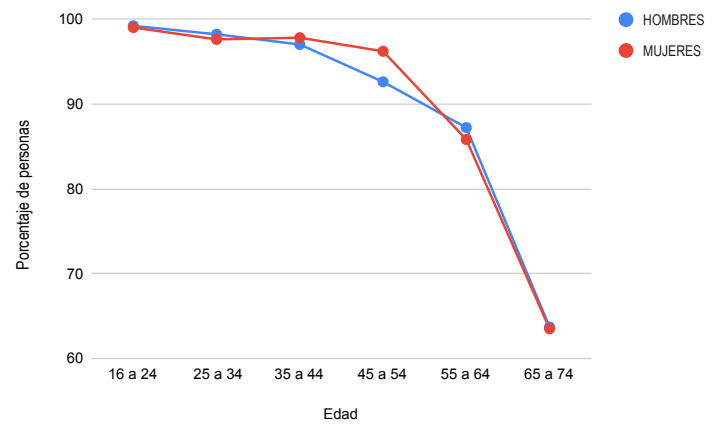

Figura 1: Datos del Instituto Nacional de Estadística sobre el uso de Internet en el cuarto trimestre del año 2019 en España [7].

En el presente artículo se describe el desarrollo de una funcionalidad destinada al robot social Mini que ofrecerá una aplicación accesible con el fin de ayudar a las personas mayores a ser más independientes y fortalecer sus habilidades con las nuevas tecnologías. Se pretende ofrecer al usuario una aplicación de alta funcionalidad que permita su uso de manera sencilla. Tras investigar que uno de los principales atractivos de las personas mayores en el uso de los dispositivos es la capacidad de comunicarse con sus seres queridos [1] (ver Fig. 2), se decide implementar una interfaz sencilla que facilite las comunicaciones con sus familiares de manera natural utilizando como plataforma WhatsApp, una de las aplicaciones más usadas en el mercado de los dispositivos [17]. El desarrollo de esta aplicación junto al robot social Mini proporciona que las personas de avanzada edad se encuentren incluidas en la sociedad actual y tengan la posibilidad de establecer lazos sociales y emocionales con sus seres queridos.

El artículo se estructura de la siguiente manera. En la Sección 2 se realiza una revisión del estado del arte donde se exponen los trabajos rela- 

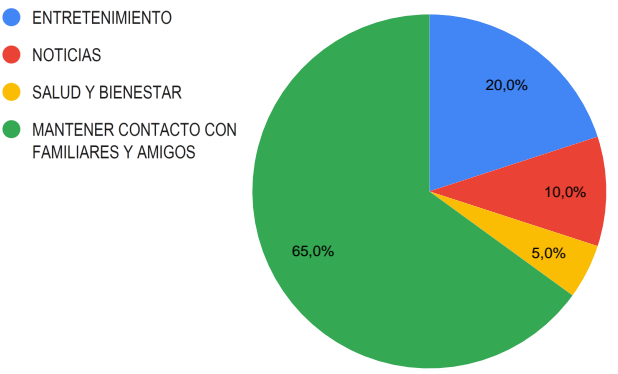

Figura 2: Estudio realizado a personas de avanzada edad sobre la utilidad de las aplicaciones móviles $[1]$.

cionados con este artículo. Posteriormente, en la Sección 3 se expone la plataforma donde se implementa el trabajo desarrollado. A continuación, en la Sección 4 se describe nuestra propuesta, la Sección 5 recoge los resultados obtenidos a partir de las opiniones de los usuarios y para finalizar, se realiza una conclusión final en la Sección 6 .

\section{ESTADO DEL ARTE}

Las familias en las sociedades modernas están separadas en espacio y tiempo. Actualmente, los familiares jóvenes viven en un mundo conectado mediante los dispositivos tecnológicos y las redes sociales. Sin embargo, los miembros mayores poseen menor inclusión social lo que conduce a un aumento de aislamiento y depresión, poniendo en riesgo su integridad física y mental [16]. Una inclusión participativa en las nuevas tecnologías puede mejorar la calidad de vida de estas personas. Para paliar esta situación, existen diversas soluciones tecnológicas, tales como robots sociales o aplicaciones accesibles.

\subsection{APLICACIONES ACCESIBLES}

La industria tecnológica está empezando a incluir entre sus productos y servicios una oferta adaptada a los perfiles de las personas de avanzada edad permitiendo su fácil asimilación, uso y aplicación. Actualmente, aparte de la funcionalidad de accesibilidad que poseen los teléfonos inteligentes y las tabletas digitales desde su fabricación, existe una creciente oferta de aplicaciones que simplifican el uso de los dispositivos haciéndolos más accesibles y sencillos mediante la disminución de funciones y la aparición de iconos y letras de mayor tamaño [18]. Estas aplicaciones se pueden clasificar en relación al tema al que se dedican:

- Relacionadas con la salud: se centran en dar diferentes servicios relacionados con la salud como pueden ser: AlzNav (aplicación que indica el camino a casa), Glucose Buddy ${ }^{1}$ (registra los datos relacionados con la glucosa), Yana (asistente psicológica, realiza diversas pautas para la disminución de ansiedad o estrés) o MediSafe (gestiona los medicamentos), entre otras [14].

- Relacionadas con el ocio: proporcionan una variedad de actividades o juegos. Como puede ser: Luminosity (plataforma multijuegos) o MemoryTrainer (actividades para la mente) [1].

- Relacionadas con la accesibilidad: se encargan de establecer un tema de dispositivo más sencillo (Wiser O BigLauncher ${ }^{2}$ ) o recondicionan los dispositivos para personas con problemas auditivos (Booster) o visuales (DragonDicatacion) [1].

\section{2. $\quad$ ROBOTS SOCIALES}

La robótica social está introduciéndose a un ritmo moderado en la sociedad permitiendo la Interacción Humano-Robot (HRI) a mayor escala [11]. Está diseñada para interactuar con las personas de manera natural e interpersonal logrando resultados positivos en ámbitos de entretenimiento, ocio o educación. En términos básicos, se puede definir como: "el robot que interactúa y se comunica con las personas (de forma sencilla y agradable) siguiendo comportamientos, patrones y normas sociales" [11]. Para ello, se dotan de capacidades similares a las que utilizan las personas para poder relacionarse utilizando una amplia gama de habilidades cognitivas [4]. De esta manera, pueden conseguir captar la atención de las personas y ser percibidos como "compañeros" que emplean el habla y acciones no verbales para expresar una idea y conseguir que el receptor interactúe de manera amigable.

En la actualidad, existen gran variedad de robots asistenciales que apoyan a las personas mayores a realizar sus actividades básicas diarias. Brindan actividades de atención como ayudar a recordar la medicación, de entretenimiento, de seguridad y de compañía. Además, algunos incorporan plataformas de comunicación propias que permiten al usuario contactar con sus seres queridos. Por último, tienen el objetivo de mejorar la calidad de vida de las personas mayores monitorizando su deterioro físico y cognitivo. Como pueden ser: MoveCare [3], Care-O-Bot ${ }^{3}$ o Mylo ${ }^{4}$.

\footnotetext{
${ }^{1}$ https://www.glucosebuddy.com/

${ }^{2}$ https://biglauncher.com/

${ }^{3}$ https://www . care-o-bot.de

${ }^{4}$ https://www. heymylo.ie/
} 


\subsection{APLICACIÓN WHATSAPP}

Actualmente, WhatsApp es la aplicación de mensajería instantánea más utilizada en el mundo, posee 2000 millones de usuarios [17]. Proporciona, sin ningún coste, enviar o recibir mensajes y realizar videollamadas o llamadas entre sus usuarios. A lo largo de su historia se han desarrollado diferentes aplicaciones basadas en su interfaz [10], como pueden ser: WhatsApp Tally ${ }^{5}$ o KyteApp ${ }^{6}$ (envían facturas, tickets o promociones de las empresas a clientes) o TwiLio $^{7}$ (permite realizar chats programados para responder a preguntas de clientes).

\section{DESCRIPCIÓN DE LA PLATAFORMA}

La aplicación se ha implementado en el robot social Mini (ver Fig. 3), proyecto desarrollado por el departamento de robots sociales de la Universidad Carlos III de Madrid cuyo propósito principal es ayudar y entretener a las personas de avanzada edad [13].

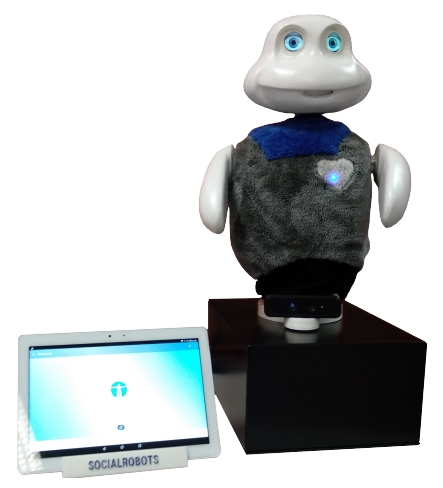

Figura 3: Robot social Mini [8].

Se trata de un robot de sobremesa amigable que cuenta con una serie de componentes que facilitan la interacción humano-robot. Posee una tableta digital donde muestra el contenido multimedia al usuario. Se encuentra recubierto de un material suave para establecer un aspecto amigable. Puede moverse y realizar gestos expresivos gracias a sus cinco grados de libertad proporcionados por los servomotores ubicados en sus articulaciones. Está equipado con un micrófono y varios altavoces que establecen la comunicación mediante la voz e incluye sensores táctiles en los hombros y vientre que permiten la interacción física por parte del usuario. Para extraer información visual y de profundidad del entorno cuenta con una serie de cámaras. Además, para representar emociones

\footnotetext{
${ }^{5}$ https://tally2whatsapp.in

${ }^{6}$ https : //www. kyteapp.com

${ }^{7}$ https://www.twilio.com/whatsapp
}

y rasgos incorpora LEDS en la boca, mejillas y corazón. Por último, en los ojos contiene pantallas uOLED que muestran GIF, permitiendo al robot mirar en diferentes direcciones y cambiar de expresión para transmitir diversos estados de ánimo [12]. Todo este hardware dota a Mini de la capacidad de interacción multimodal para establecer la comunicación de forma natural con el usuario. $\mathrm{Su}$ estructura software se compone de diferentes sistemas representados en la Fig. 4.

- Sistema de toma de decisión (DMS): encargado de ejecutar y controlar cada aplicación. Selecciona la más adecuada dependiendo del usuario [8].

- Sistema de interacción robot - usuario (HRI): administra los mensajes procedentes del DMS y de las aplicaciones para enviar la información necesaria a los paquetes que intervienen en la interacción con el usuario mediante la generación de audio (TTS) y reconocimiento de voz (ASR) [6].

- Servidor de parámetros: obtiene la información del usuario de un servidor remoto.

- Aplicaciones: diferentes habilidades que dotan al robot de la posibilidad de ofrecer ocio, entretenimiento [15], terapias o contenido multimedia.

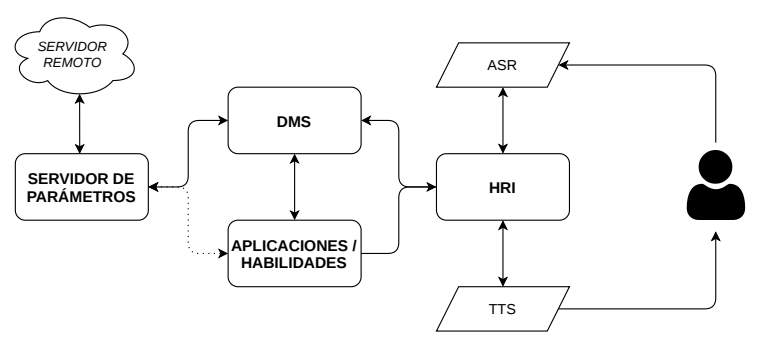

Figura 4: Diagrama de la estructura software robot Mini.

\section{NUESTRA PROPUESTA}

El presente artículo elimina la fricción que poseen las personas mayores a la hora de utilizar las redes sociales para comunicarse con sus seres queridos. Brinda la oportunidad de emplear WhatsApp de manera sencilla e intuitiva con el robot social Mini manteniendo una comunicación natural. Corresponde con una serie de módulos que permiten la conexión con la plataforma para conseguir el uso más frecuente de esta tecnología.

Además con esta aplicación, se pretende iniciar una nueva línea de desarrollo (Android-ROS ${ }^{8}$ ) pa-

${ }^{8}$ http://wiki.ros.org/android 
ra el robot social Mini que permita una mejora en las interfaces con el usuario prestando distintos servicios. De esta manera, se busca desarrollar diversas aplicaciones más accesibles e intuitivas ofreciendo al usuario una aplicación más dinámica guiada por el robot social Mini. Por tanto, se brinda la ventaja de utilizar el robot Mini como un robot social de servicios frente a las aplicaciones convencionales que se encuentran en los dispositivos móviles.

\subsection{CARACTERÍSTICAS GENERALES}

En esta sección se define el marco de desarrollo software utilizado. El programa se desarrolla bajo el subproyecto android_tutorial_pubsub procedente del proyecto android-core, oficial de AndroidROS. Este repositorio proporciona las librerías y bibliotecas necesarias para realizar las conexiones bidireccionales entre el robot y la aplicación.

La aplicación se estructura a partir de dos módulos: Módulo de Android y Módulo de ROS (Robot Operating System). La comunicación entre ambos se efectúa mediante el paso de mensajes que permite realizar suscripciones y publicaciones a diferentes topics desde los nodos creados, esto proporciona la conexión con los módulos del robot. La plataforma de Android ofrece el desarrollo de la interfaz gráfica de la aplicación para proporcionar al usuario la conexión con WhatsApp de manera sencilla e intuitiva.

En líneas paralelas al hilo principal, se encuentran los servicios, activos durante todo el ciclo de ejecución. Los implementados en la aplicación sirven para: recoger información fuera del entorno de la aplicación, crear un sistema de comunicación con ROS durante todo el ciclo de ejecución y acceder a la aplicación WhatsApp con la accesibilidad que aporta Android en sus dispositivos.

\subsection{REQUISITOS PREVIOS}

Es necesario realizar una configuración previa en la tableta digital que posee Mini. Para que la aplicación desarrollada funcione de manera correcta y obtenga todas las funcionalidades.

Es imprescindible la presencia de la aplicación en el dispositivo Android. No disponer de ella impedirá el uso de la aplicación desarrollada. Asimismo, se requiere una cuenta con un número de teléfono no utilizado en otro dispositivo, debido a que WhatsApp no permite el registro de un mismo número en diferentes dispositivos electrónicos.

Asimismo, es necesario establecer los permisos de acceso a los contactos del dispositivo para poder realizar llamadas y videollamadas. De igual manera, hay que otorgar el permiso de acceso de las notificaciones para la posterior lectura de los mensajes entrantes. Por último, se debe permitir el servicio de accesibilidad proporcionado por Android para poder enviar los mensajes automáticamente (ver Sección 4.4).

\subsection{DISEÑO DE LA INTERFAZ DEL USUARIO}

La aplicación, como se ha comentado en la sección 4.1, está formada por diversos módulos que permiten tanto la conexión con el robot como con la aplicación de WhatsApp. Brinda la capacidad de recibir y contestar a los mensajes entrantes, realizar llamadas o videollamadas y enviar mensajes. La interfaz ofrece al usuario tres tipos de pantalla intuitivas y accesibles.

La pantalla principal (ver Fig. 5) consta de cuatro botones cuyo objetivo es redirigir al usuario a la actividad deseada. Cada botón se diseña con un color que contiene el nombre e icono representativo de cada actividad. El funcionamiento del menú se detalla en la Sección 4.4.

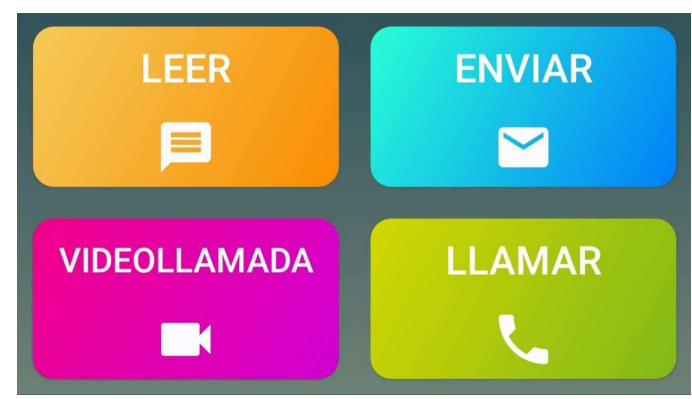

Figura 5: Diseño gráfico de la pantalla principal.

La pantalla de contactos (ver Fig. 6) recoge una matriz $3 \times 2$ donde cada posición corresponde a un contacto. A lo largo de la realización del proyecto se han llevado a cabo varias encuestas a personas de avanzada edad para determinar un número aproximado de contactos favoritos. Asimismo, se ha observado que la aparición en pantalla de muchos contactos en vista de lista es peor que una selección de varios contactos agrupados en una matriz. Además, para proporcionar al usuario una visión intuitiva de sus contactos favoritos a cada botón se le establece un color. Esta pantalla aparece cuando el usuario decide realizar una videollamada, llamada o enviar mensajes, su funcionamiento se detalla en la Sección 4.4.

Muchas de las personas mayores sienten dificultad a la hora de leer texto en las pantallas de los dispositivos electrónicos. Por ello, la pantalla de lectura de mensajes (ver Fig. 8) representa los mensajes 


\begin{tabular}{|l|l|l|}
\hline MARIA & FELIX & ESTELA \\
\hline MIGUEL & SARA & DANIEL \\
\hline 4 & ATRÁs \\
\hline
\end{tabular}

Figura 6: Diseño gráfico del fragmento contactos.

entrantes de manera separada en una lista con un tamaño de letra mayor y sin entrar directamente a la pantalla principal de la conversación de WhatsApp. Asimismo, si el usuario lo desea el robot puede efectuar la lectura en alto de los mensajes entrantes. El funcionamiento de esta pantalla se detalla en la siguiente Sección.

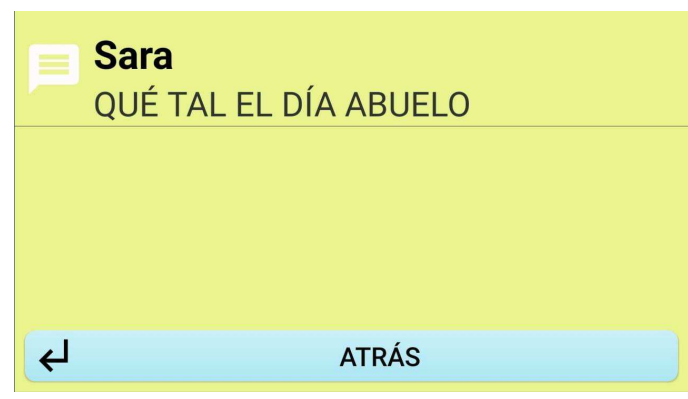

Figura 7: Diseño gráfico del fragmento leer.

\subsection{FUNCIONALIDAD}

La funcionalidad de la aplicación desarrollada se divide en distintas partes que necesitan de su interrelación para el correcto funcionamiento.

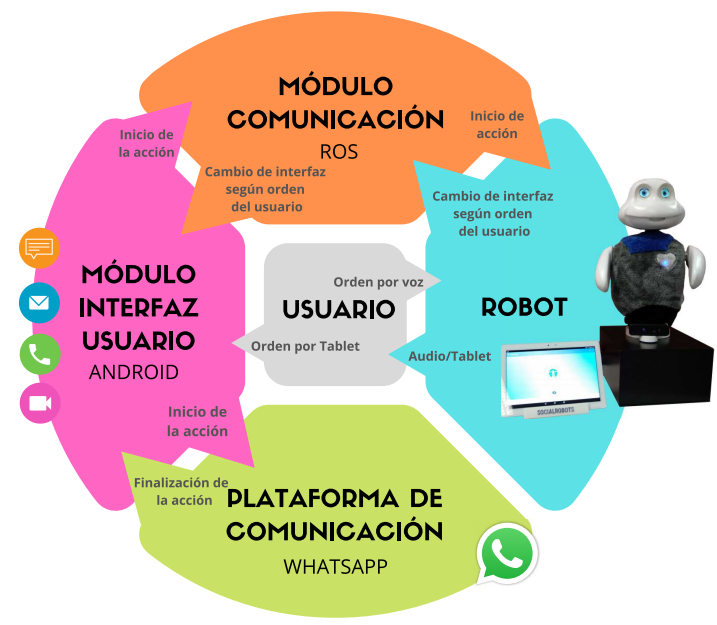

Figura 8: Esquema de los principales módulos de la aplicación y sus relaciones.
- Módulo de comunicación: se realiza a través de ROS, permite la comunicación bidireccional entre el robot y la aplicación. Es el encargado de conectarse a los diferentes módulos del robot, comentados en el Capítulo 3. Está relacionado con el módulo de interacción con el usuario, permite al robot controlar las acciones de la aplicación según la necesidad determinada. Por tanto, facilita al usuario el uso de la aplicación realizando la mínima interacción con la pantalla de la tableta digital.

- Funcionalidad de interacción: por un lado, se encarga de recoger la participación del usuario tanto por voz o por toques de pantalla. Se utiliza el ASR del robot para brindar mayor accesibilidad al usuario, esta interacción se denomina humano-robot. Por otro lado, se presenta la interacción robot-humano donde el robot es capaz de guiar mediante el ETTS al usuario dependiendo de la actividad.

- Módulo de permisos: con el objetivo de cumplir las leyes de Protección de Datos y facilitar la configuración de la aplicación. En el primer uso cuenta con un módulo de permisos donde aparecen diferentes ventanas emergentes que permiten al usuario activarlos de manera sencilla y con una breve explicación.

- Funcionalidad de leer mensajes: se encuentra ligado con la pantalla de la Fig. 8. En primer lugar, mediante una ventana emergente permite al usuario decidir si desea la lectura del mensaje en voz alta. Este fragmento está compuesto de una lista que ofrece una visión simplificada de los mensajes entrantes del WhatsApp. Para ello, es necesario suscribirse a las notificaciones mediante un servicio de acceso. Una vez leídos los mensajes, se proporciona al usuario la oportunidad de contestarlos mediante la transcripción de voz a texto, ver Funcionalidad de enviar mensajes. Es importante mencionar que la entrada de mensajes se realiza de todos los contactos para sacar máxima rentabilidad de manera sencilla a la presente aplicación.

- Funcionalidad de enviar mensajes: la pantalla correspondiente a este módulo es la representada en la Fig. 6. Permite escribir mensajes a los contactos favoritos mediante la transcripción de voz a texto. Una vez recogido el mensaje lo envía de manera automática a la conversación correspondiente.

- Funcionalidad de realizar llamadas y videollamadas: ambos módulos contienen el 
mismo funcionamiento excepto la petición final donde se decide si se realiza una llamada o videollamada. Están ligados a la Fig. 6 donde permite al usuario escoger el contacto, posteriormente se busca el contacto en la agenda del dispositivo y se realiza la acción correspondiente.

- Funcionalidad salir: permite al usuario finalizar la aplicación. Para ello, debe encontrarse en el menú principal e indicar mediante voz al robot que desea salir de la aplicación.

\section{RESULTADOS}

Para verificar el uso de la aplicación se proporciona la aplicación desarrollada a un grupo de residentes de la residencia Virgen de la Nueva. Los usuarios indican que a pesar de poseer un teléfono inteligente no utilizan de manera habitual WhatsApp debido a su dificultad a la hora de buscar a los contactos, de escribir por el teclado y/o de leer los mensajes entrantes. Además, muestran su agradecimiento debido al acercamiento de las Nuevas Tecnologías e indican que la experiencia ha sido agradable al ser ellos mismos los que manejan la aplicación, con ayuda del robot, para poder conectarse con sus familiares de manera autónoma (ver Fig. 9).

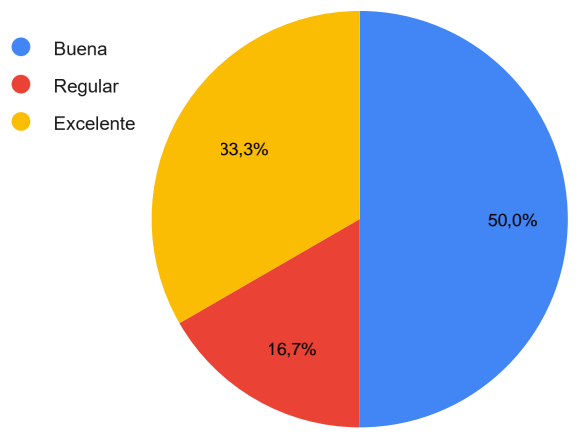

Figura 9: Experiencia de los usuarios encuestados a la hora de utilizar el robot social Mini con la aplicación desarrollada.

\section{CONCLUSIONES}

Este artículo se enfoca en el desarrollo de aplicaciones con alta funcionalidad que permitan a las personas de edad avanzada reforzar el uso de las TIC. En concreto, acerca las redes sociales a este colectivo. Gracias al robot social Mini se consigue eliminar las fricciones que sufren estas personas a la hora de utilizar los dispositivos electrónicos. El robot social Mini en este desarrollo utiliza sus habilidades de interacción humano-robot para guiar al usuario y utilizar la plataforma WhatsApp de manera sencilla y natural. Se proporciona al usuario la capacidad de interactuar de dos maneras diferentes, mediante la tableta digital o por voz, para facilitar el uso de la aplicación.

Teniendo en cuenta la satisfacción de los usuarios de la residencia Virgen de la Nueva se puede concluir que se ha conseguido un desarrollo software adecuado y accesible para personas mayores. Es importante mencionar que los usuarios se han sentido agradecidos por la facilidad que brinda el robot social Mini para incluirlos en las Nuevas Tecnologías de la Información proporcionándoles la capacidad de comunicarse con sus seres queridos de manera autónoma sin necesidad de terceras personas. Además, han contemplado a esta plataforma como un "compañero/amigo" que ofrece diversas actividades, como pueden ser: contenido multimedia o sesiones de tratamiento específicas.

Asimismo, esta aplicación se propone como base para futuros desarrollos en el robot Mini, debido a su capacidad de diseño que permite la creación de aplicaciones de alta funcionalidad con estilo sencillo e intuitivo para reforzar el uso de las TIC en las personas de avanzada edad.

\section{Agradecimientos}

La investigación desarrollada ha recibido financiación de dos proyectos: "Development of social robots to help seniors with cognitive impairment" (ROBSEN), financiado por el Ministerio de Economía y Competitividad; y "RoboCity2030IIICM", financiado por la Comunidad de Madrid y cofinanciado por los Fondos Estructurales de la Unión Europea.

\section{English summary}

REMOTE COMMUNICATION
BETWEEN FAMILY MEMBERS
THROUGH SOCIAL ROBOTICS

\begin{abstract}
Nowadays, a large number of senior people do not make frequent use of new information technologies. In this work, we try to eliminate the friction that they have to communicate with their family and friends naturally through technological means. We propose an accessible and intuitive communication application implemented with the Mini social robot. We developed a graphical interface that allows users to have conversations, calls, and video calls through a
\end{abstract}


simple interface via Mini robot. The communication platform used is WhatsApp, one of the most widely used applications on the smart device market today. The aim is to contribute to the care and well-being of the elderly.'

Keywords: Social robotics; Human-robot interaction; Communication through social robotics; Digital inclusion; Inclusive social networks.

\section{Referencias}

[1] L. Abad, «Media literacy for older people facing the digital divide: The e-inclusion programmes design,» Revista científica iberoamericana de comunicación y educación, vol. 21, n. ${ }^{\circ}$ 42, pp. 173-180, 2014.

[2] L. Abad y A. P. Agregado, «La alfabetización digital como instrumento de e-inclusión de las personas mayores,» Prisma Social, vol. 16, pp. 156-204, junio-noviembre 2016.

[3] N. Alberto Borghese y C. Galán de Isla, «Cuidador virtual inteligente MoveCare Paraninfo Digital,» vol. XIII, n. ${ }^{\circ}$ 30, 2019.

[4] C. Breazeal, K. Dautenhahn y T. Kanda, «Social robotics,» en Springer Handbook of Robotics, Springer International Publishing, 2016, pp. 1935-1971.

[5] R. Casado-Muñoz, F. Lezcano y M. J. Rodríguez-Conde, "Active ageing and access to technology: An evolving empirical study,»Revista Cientifica de Educomunicación, vol. XXIII, n. ${ }^{\circ}$ 45, pp. 37-46, 2015.

[6] E. Fernández-Rodicio, Á. Castro-González, F. Alonso-Martín, M. Maroto-Gómez y M. Salichs, «Modelling multimodal dialogues for social robots using communicative acts,» Sensors (Switzerland), vol. 20, n. ${ }^{\circ} 12$, pp. 1-30, 2020.

[7] Instituto Nacional de Estadistica, Instituto Nacional de Estadística. Productos y Servicios, 2017. [En línea]. Disponible en: https: //bit.1y/2ZffBBk (Acceso: 15-06-2021).

[8] M. Maroto-Gómez, Á. Castro-González, J. C. Castillo, M. Malfaz y M. A. Salichs, « bio-inspired motivational decision making system for social robots based on the perception of the user,» Sensors (Switzerland), vol. 18, n. ${ }^{\circ} 8,2018$.
[9] X. Martínez-Rolán y T. Piñero-Otero, «Tipología y funcionalidades de las aplicaciones móviles para mayores. A un tap del envejecimiento activo,» Ámbitos. Revista Internacional de Comunicación, vol. 0, n. ${ }^{\circ}$ 29, 2015.

[10] P. Mehta y R. Sandhu, «Whatsapp Web Reverse Engineering,» pp. 1-41, 2019. [En línea]. Disponible en: http : / / 172 . 16. 73 . 104 : $8080 /$ jspui/handle/123456789/ 22743.

[11] A. J. Pérez Vidal, Castro-González, F. Alonso Martín, J. C. Castillo y M. Salichs, «Evolución de la robótica social y nuevas tendencias,» en Actas de las XXXVIII Jornadas de Automática, Gijón, 2017, pp. 836-843. [En línea]. Disponible en: https : / / doi .org / 10.17979/spudc.9788497497749.0836.

[12] E. Salichs, E. Fernández-Rodicio, J. C. Castillo, Á. Castro-González, M. Malfaz y M. Á. Salichs, «A social robot assisting in cognitive stimulation therapy,» en Advances in Practical Applications of Agents, Multi-Agent Systems, and Complexity: The PAAMS Collection. PAAMS 2018. Lecture Notes in Computer Science, vol. 10978, Springer, Cham, 2018, pp. 344-347. [En línea]. Disponible en: https : / /doi .org/10 . 1007/978-3-31994580-4_35.

[13] M. A. Salichs, Á. Castro-González, E. Salichs, E. Fernández-Rodicio, M. MarotoGómez, J. J. Gamboa-Montero, S. MarquesVillarroya, J. C. Castillo, F. Alonso-Martín y M. Malfaz, «Mini: A New Social Robot for the Elderly,» International Journal of Social Robotics, vol. 12, n. ${ }^{\circ}$ 6, pp. 1231-1249, 2020. [En línea]. Disponible en: https : / / doi . org/10.1007/s12369-020-00687-0.

[14] M. Sánchez López, J. Fernández Alemán, A. Toval y J. Carrillo De Gea, «Teléfonos inteligentes para Smart Phones for the elderly: la tercera edad: una revisión de a review of mobile aplicaciones móviles de salud health applications,» Rev Costarr Salud Pública, vol. 24, pp. 30-42, 2014.

[15] E. Velázquez Navarro, S. González-Díaz, F. Alonso-Martín, J. Castillo, CastroGonzález, M. Malfaz y M. Salichs, «El robot social Mini como plataforma para el desarrollo de juegos de interacción multimodales,» jun. de 2019.

[16] J. Waterworth, «Diseño de Sistemas y Herramientas TIC Orientados a las Necesidades Psicológicas y Sociales de las Personas Mayores que Viven de Forma Independiente,» RevistaeSalud.com, vol. 7, n. ${ }^{\circ} 26$, pp. 43-9, 2011. 
[17] WhatsApp, Acerca de WhatsApp, 2020. [En línea]. Disponible en: https : / / www . whatsapp . com / about/ (Acceso: 19-06-2021).

[18] L. A. Álvarez Álvarez, «Mayores en la era internet. Nuevos sistemas y aplicaciones prácticas,» XVIII Jornadas sobre Asociacionismo en los Programas Universitarios de Mayores, 2019.

(C) $\left(20\right.$ (2) $\begin{array}{l}\text { C } 2021 \text { by the authors. } \\ \text { Submitted for possible } \\ \text { open access publication }\end{array}$ under the terms and conditions of the Creative Commons Attribution CC BY-NC-SA 4.0 license (https://creativecommons.org/licenses/by-ncsa/4.0/deed.es). 